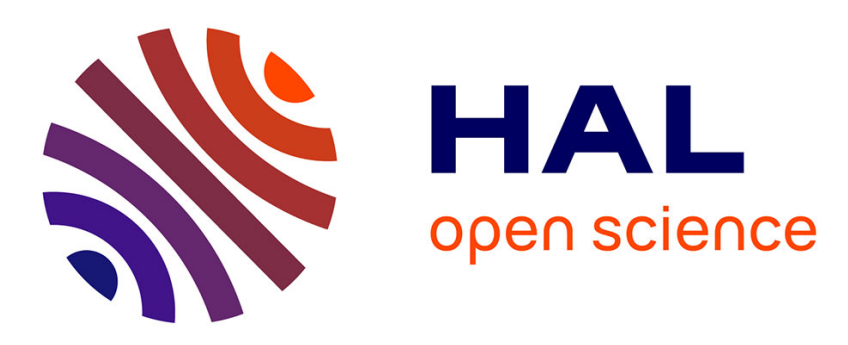

\title{
Nonperturbative chemical modification of graphene for protein micropatterning
}

Vamsi K. Kodali, Jan Scrimgeour, Suenne Kim, John H. Hankinson, Keith M. Carroll, Walt A de Heer, Claire Berger, Jennifer E. Curtis

\section{- To cite this version:}

Vamsi K. Kodali, Jan Scrimgeour, Suenne Kim, John H. Hankinson, Keith M. Carroll, et al.. Nonperturbative chemical modification of graphene for protein micropatterning. Langmuir, 2011, 27 (3), pp.863. 10.1021/la1033178 . hal-01002906

\section{HAL Id: hal-01002906 https://hal.science/hal-01002906}

Submitted on 7 Jun 2014

HAL is a multi-disciplinary open access archive for the deposit and dissemination of scientific research documents, whether they are published or not. The documents may come from teaching and research institutions in France or abroad, or from public or private research centers.
L'archive ouverte pluridisciplinaire HAL, est destinée au dépôt et à la diffusion de documents scientifiques de niveau recherche, publiés ou non, émanant des établissements d'enseignement et de recherche français ou étrangers, des laboratoires publics ou privés. 


\title{
Nonperturbative Chemical Modification of Graphene for Protein Micropatterning
}

Vamsi K. Kodali, $\uparrow, \uparrow$ Jan Scrimgeour, $\uparrow, \uparrow$ Suenne Kim,$\uparrow$ John H. Hankinson, $\uparrow$ Keith M. Carroll, $, \uparrow, \uparrow$

Walt A. de Heer, $\uparrow$ Claire Berger, $\dagger, \S$ and Jennifer E. Curtis* $, \dagger, \dagger$

$\dagger$ School of Physics, and $\ddagger$ Parker H. Petit Institute for Bioengineering and Biosciences, Georgia Institute of Technology, 837 State Street, Atlanta, Georgia 30332-0430, United States, §CNRS-Institut N_eel, BP 166, 38042 Grenoble, Cedex 9, France

\begin{abstract}
Graphene's extraordinary physical properties and its planar geometry make it an ideal candidate for a wide array of applications, many of which require controlled chemical modification and the spatial organization of molecules on its surface. In particular, the ability to functionalize and micropattern graphene with proteins is relevant to bioscience applications such as biomolecular sensors, single-cell sensors, and tissue engineering.Wereport a general strategy for the noncovalent chemical modification of epitaxial graphene for protein immobilization and micropatterning. We show that bifunctional molecule pyrenebutanoic acidsuccinimidyl ester (PYR-NHS), composed of the hydrophobic pyrene and the reactive succinimide ester group, binds to graphene noncovalently but irreversibly. We investigate whether the chemical treatment perturbs the electronic band structure of graphene using X-ray photoemission (XPS) and Raman spectroscopy. Our results show that the sp2 hybridization remains intact and that the $\pi$ band maintains its characteristic Lorentzian shape in the Raman spectra. The modified graphene surfaces, which bind specifically to amines in proteins, are micropatterned with arrays of fluorescently labeled proteins that are relevant to glucose sensors (glucose oxidase) and cell sensor and tissue engineering applications (laminin).
\end{abstract}


The extraordinary physical properties of graphene and its planar geometry make graphene an ideal candidate for many device technologies. 1 This includes applications in the bioscience arena where closely related carbon nanotube devices have already been used for biomolecular sensors, 2 single-cell sensors, 3 and tissue engineering.4,5 Recent developments have enabled the fabrication of extremely pure, large-area graphene samples that extend over millimeters in the form of epitaxial graphene (EG).6 Standard fabrication techniques can be used to pattern graphene lithographically, 7,8 permitting a degree of control and versatility not accessible to the potential devices constructed from graphene's counterpart, the carbon nanotube. The controlled chemical and spatial assembly of molecules on the surface of graphene will be a key element in future optimized graphene devices. Many studies report tuning the electronic properties of graphene using chemical,9,10 lithographic,11 and external field approaches.12 Fewer efforts focus on the functionalization and patterning of EG for the specific self-assembly of biomolecules or other objects such as nanoparticles. 13 In this letter, we establish a simple approach to functionalizing graphene non covalently for subsequent immobilization and micrometer-resolution spatial patterning of proteins (Figure 1). The chemical scheme presented here allows for robust, spatially resolved protein anchoring to the surface and has the important feature that it does not perturb the desirable electronic properties of graphene

The chemical modification of graphene for protein crosslinking is achieved using bifunctional molecule 1pyrenebutanoic acid succinimidyl ester (PYR-NHS) (Anaspec, Inc. USA). The aromatic pyrenyl group in PYR-NHS interacts strongly with the basal plane of graphene via $\pi$ stacking, 14 without perturbing the sp2 bond structure of the carbon honeycomb lattice, or the $\pi$ band responsible for graphene's characteristic electronic properties. This is confirmed here using X-ray photoelectron spectroscopy (XPS) and Raman spectroscopy. We subsequently demonstrate that proteins can be precisely anchored via the succinimidyl ester group and patterned on graphene with micrometer-scale resolution. All graphene samples used in this work were produced following well-established protocols6 and consist of epitaxial graphene (EG) grown on the $\mathrm{C}$ face of $4 \mathrm{H}$ silicon carbide in an induction furnace. Each sample has a surface area of $3.5 \times 4.5$ $\mathrm{mm} 2$ and is about three atomic layers thick, as verified by ellipsometry.

The irreversible binding of PYR-NHS to the epitaxial graphene is critical to ensuring the immobilization of proteins onto the surface. Because the PYR-NHS interaction is noncovalent, the robustness of the attachment was confirmed using XPS. The innate hydrophobicity of both graphene and PYR-NHS is expected to facilitate the adsorption of the molecule fromsolution to the graphene surface and make it inherently stable against desorption under aqueous conditions. Graphene samples were incubated in PYRNHS (6 mM in dimethylformamide (DMF)) for $1 \mathrm{~h}$ at room temperature and then thoroughly rinsed three times with DMF. The comparison of spectra from pristine EG and PYR-EG shows little difference. To increase the signal corresponding to PYR-NHS, it was reacted, after incubation with EG, with fluorinated molecule $1 \mathbf{H}, 1 \mathrm{H}$-perfluorooctylamine (PFOA, incubation for $1 \mathrm{~h}$ followed by rinsing with DMF and then with DI water). The recorded spectrum (Figure 2) shows a strong fluorine F 1s peak. Controls demonstrate that the nonspecific binding of PFOA to EG is negligible, with a minimal signal in F 1s (Figure 2a). Hence, even after harsh washing, XPS confirms that strong interactions of PYR with graphene anchor the molecules to the surface.

Measurements at multiple points on the same sample consistently showed F 1s signals for the PYR-PFOA complexes, indicating that PYR covers EGrelatively uniformly. The successful binding of PFOAonly in the presence of PYR-NHS also demonstrates the flexibility of the molecule in cross-linking amines, indicating that awide range of proteins and other nanoobjects can in principle be bound to the graphene.

The chemical modification of graphene can change its band structure, an approach that is currently of great interest for tailoring the electronic properties of graphene. For future applications in molecular and cell biosensing, however, it may be desirable to maintain graphene's highly sensitive conductive nature after the chemical preparation of the surface with an NHS cross-linker. Here,we showthat the noncovalent interaction of PYRwith EGdoes not disrupt graphene's sp2 hybridization or perturb its $\pi$-band structure. Raman spectroscopy of PYRtreated graphene shows an absence of theD peak that is expected to arise at $\sim 1350 \mathrm{~cm}-1$ with the formation of sp3 structure (Figure 2b). 15 This observation is corroborated by XPS measurements that show that the $\mathrm{C}$ 1s peak corresponding to $\mathrm{sp} 2$ hybridization remains unmodified after PYR treatment (SI). Previous XPS studies demonstrated that the transformation of carbon centers from $\mathrm{sp} 2$ to sp3 changes the $\mathrm{C} 1 \mathrm{~s}$ peak to a broad envelope, which is not observed here. 9 
The Raman data also show a 2D Lorentzian peak at $\sim 2716 \mathrm{~cm}-1$ before and after the PYR treatment. A single Lorentzian peak at this wavenumber is consistent with an unperturbed $\pi$ band, indicating that graphene's key electronic properties remain intact after its chemical functionalization with PYR.16,17

The immobilization of proteins onto graphene is achieved by reacting the NHS group on the graphenebound PYR with the amines in the lysine residues present in most proteins. The same strategy has been demonstrated previously on graphite 18,19 and carbon nanotubes. 14

Micrometer-resolution spatial patterning of proteins onto graphene was accomplished using microcontact printing.20 Figure 1a shows a fluorescent image of PYR-treated EG micropatternedwith glucose oxidase.Glucose oxidase is a small $144 \mathrm{kDa}$ enzyme and a critical component of electrochemical glucose biosensor designs.21 Graphene-based glucose biosensors should be extremely sensitive to minute concentrations of glucose, similar to the sensitivity of carbon nanotube glucose sensors.2,3 Microcontact printingwas achieved using a PDMS stamp incubated for $15 \mathrm{~min}$ with fluorescently labeled glucose oxidase in solution (Supporting Information). The resultant large-scalemicropattern has rings of circular microareas approximately $\sim 5.5 \mu \mathrm{m}$ in diameter (Figure $2 \mathrm{a}$ ). Similar patterns have also been produced on non-PYRtreated graphene via nonspecific binding; however, for many applications, the stability of the immobilized protein is critical, making irreversible binding using PYR-NHS a valuable alternative.

To measure the typical height of the printed protein areas, the PYR-treated graphene was imaged with atomic forcemicroscopy (AFM). The topographical images were collected using an Agilent 5600 LS working in contact mode in liquid at a scan rate of $0.3 \mathrm{~Hz}$ using a silicon tip coated with $\mathrm{Cr}$ and $\mathrm{Au}$ with a small spring constant of $\sim 3.5 \mathrm{~N} / \mathrm{m}$. Figure 3 shows a line profile of a surface printed with glucose oxidase using a stamp with 2.5- $\mu$ mdiameter circles spaced $\sim 5 \mu \mathrm{m}$ apart. Each of the three regions' profiles has an average height of between 4 and $8 \mathrm{~nm}$. This is consistent with the molecular weight of glucose oxidase, which has a hydrodynamic radius of $4.3 \mathrm{~nm}, 22$ as well as with electron microscopy studies that have shown that it has dimensions of $5 \mathrm{~nm} \_8 \mathrm{~nm} .23$ Hence, the bound protein likely constitutes a monolayer.

The detection of the fluorescently labeled proteins on the surface of graphene is somewhat surprising. Recent work has shown theoretically and experimentally that dyes 24,25 as well as semiconductor nanocrystals 26 are fluorescently quenched when bound to graphene. These reports include a detailed study of PYR-NHS, the aromatic dye used here, which shows that PYR is fluorescently quenched via electron transfer when covalently bound to amine-deritivatized graphene. In our studies, imaging the fluorescent patterns required much longer exposure times than for micropatterns made on glass slides (2-10 s), and the resultant images were still very dim. Further investigation is necessary to clarify whether this is due to a difference in the protein concentation, fluorescence quenching, or some other effect.

Another potential application of graphene lies in the arena of tissue engineering, where it is desirable to use combined chemical and electrical signaling to orchestrate the formation of complex cellular networks.4,5,27 We demonstrate the micropatterning of laminin, an $\sim 800 \mathrm{kDa}$ extracellular matrix protein used for neuronal guidance, on graphene. The PDMS stamp was incubated with a $100 \mu \mathrm{g} / \mathrm{mL}$ fluorescently labeled laminin solution (Trevigen, Inc., diluted in 10mMPBS atpH7.4with 5\%glycerol) and then pressed onto the graphene surface.The resultant laminin pattern is shown in Figure 1b. Micropatterning approaches such as those shown here could be used to build a massively parallel single-cell analysis device, 3,28 wheremicropatterned proteins integrated with electrical circuits would mediate the specific binding of single cells to designated areas, followed by electrical measurements to distinguish one cell type from another.

In summary, we have noncovalently functionalized epitaxial graphene, having a small number of layers, with PYR-NHS without disrupting graphene's electronic structure. Furthermore, we have demonstrated that PYR-functionalized graphene can be micropatterned with immobilized proteins using microcontact printing. The immobilization strategy used here can further be extended to attach other types of nanoobjects such as inorganic nanoparticles and synthetic polymers. The ability to dictate the location of proteins spatially with high resolution complements established lithographymethods that are currently used to control the physical layout of graphene. Future graphene-based technologies, such as massively parallel 
sensors, will benefit from spatially coordinated, high-resolution sensitive electronics and molecular patterning.

Acknowledgment. This research was supported by the NSFMRSEC through contractDMR-0820382 and theKeck Foundation. We thank E. Riedo for useful discussions.

Supporting Information Available: Experimental details of the chemical modification of graphene. XPS, Raman spectroscopy, protein preparation, and microcontact printing. This material is available free of charge via the Internet at http://pubs.acs.org. 


\section{Reference:}

(1) (a) Berger, C.; Song, Z.; Li, T.; Li, X.; Ogbazghi, A. Y.; Feng, R.; Dai, Z.; Marchenkov, A. N.; Conrad, E. H.; First, P. N.; de Heer, W. A. J. Phys. Chem. B 2004, 108, 19912. (b) de Heer, W. A.; Berger, C.; Wu, X.; Hu, Y.; Ruan, M.; Stroscio, J. A.; First, P. N.; Haddon, R.; Piot, B.; Faugeras, C.; Potemski, M.; Moon, J.-S. J. Phys. D: Appl. Phys. 2010, 37, 374007.

(2) (a) Wang, J.; Liu, G.; Jan, M. R. J. Am. Chem. Soc. 2004, 126, 3010. (b) Lin, Y.; Lu, F.; Tu, Y.; Ren, Z. Nano Lett. 2003, 4, 191-195. Besteman, K.; Lee, J.-O.; Wiertz, F. G. M.; Heering, H. A.; Dekker, C. Nano Lett. 2003, 3, 727.

(3) Heller, I.; Smaal, W. T. T.; Lemay, S. G.; Dekker, C. Small 2009, 5, 2528.

(4) Lovat, V.; Pantarotto, D.; Lagostena, L.; Cacciari, B.; Grandolfo, M.; Righi, M.; Spalluto, G.; Prato, M.; Ballerini, L. Nano Lett. 2005, 5, 1107.

(5) Mattson, M.; Haddon, R.; Rao, A. J. Mol. Neurosci. 2000, 14, 175.

(6) (a) Berger, C.; Song, Z.; Li, X.; Wu, X.; Brown, N.; Naud, C.; Mayou, D.; Li, T.; Hass, J.; Marchenkov, A. N.; Conrad, E. H.; First, P. N.; de Heer, W. A. Science 2006, 312, 1191. (b) Hass, J.; Heer, W. A.; Conrad, E. H. J. Phys.: Condens. Matter 2008, 20, 323202.

(7) Kedzierski, J.; Hsu, P.-L.; Healey, P.; Wyatt, P.; Keast, C.; Sprinkle, M.; Berger, C.; A., de Heer, W. IEEE Trans. Electron Devices 2008, 55, 2078.

(8) Moon, J. S.; Curtis, D.; Hu, M.; Wong, D.; McGuire, C.; Campbell, P. M.; Jernigan, G.; Tedesco, J. L.; VanMil, B.; Myers-Ward, R.; Eddy, C. J.; Gaskill, D. K. IEEE Electron Device Lett. 2009, 30, 650.

(9) Bekyarova, E.; Itkis, M. E.; Ramesh, P.; Berger, C.; Sprinkle, M.; de Heer, W. A.; Haddon, R. C. J. Am. Chem. Soc. 2009, 131, 1336.

(10) Wu, X.; Sprinkle, M.; Li, X.; Ming, F.; Berger, C.; de Heer, W. A. Phys. Rev. Lett. 2008, 101, 026801.

(11) Han, M. Y.; € Ozyilmaz, B.; Zhang, Y.; Kim, P. Phys. Rev. Lett. 2007, 98, 206805.

(12) Castro, E. V.; Novoselov, K. S.; Morozov, S. V.; Peres, N. M. R.; dos Santos, J. M. B. L.; Nilsson, J.; Guinea, F.; Geim, A. K.; Neto, A. H. C. Phys. Rev. Lett. 2007, 99, 216802.

(13) Wang, Q. H.; Hersam, M. C. Nat. Chem. 2009, 1, 206.

(14) Chen, R. J.; Zhang, Y.; Wang, D.; Dai, H. J. Am. Chem. Soc. 2001, 123, 3838.

(15) Lee, D. S.; Riedl, C.; Krauss, B.; von Klitzing, K.; Starke, U.; Smet, J. H. Nano Lett. $2008,8,4320$.

(16) Ferrari, A. C.; Meyer, J. C.; Scardaci, V.; Casiraghi, C.; Lazzeri, M.; Mauri, F.; Piscanec, S.; Jiang, D.; Novoselov, K. S.; Roth, S.; Geim, A. K. Phys. Rev. Lett. 2006, 97, 187401.

(17) Faugeras, C.; Nerriere, A.; Potemski, M.; Mahmood, A.; Dujardin, E.; Berger, C.; de Heer, W. A. Appl. Phys. Lett. 2008, 92, 011914.

(18) Jaegfeldt, H.; Kuwana, T.; Johansson, G. J. Am. Chem. Soc. 1983, 105, 1805.

(19) Katz, E. J. Electroanal. Chem. 1994, 365, 157.

(20) Mrksich, M.; Dike, L. E.; Tien, J.; Ingber, D. E.; Whitesides, G. M. Exp. Cell Res. 1997, $235,305$.

(21) Wang J. Chem. Rev. 2007, 108, 814-825.

(22) Heller, A. Acc. Chem. Res. 1990, 23, 128-134.

(23) Rando, D.; Kohring, G. W.; Giffhorn, F. Appl. Microbiol. Biotechnol. 1997, 48, 34-40.

(24) Ramakrishna Matte, H. S. S.; Subrahmanyam, K. S.; Venkata Rao, K.; George, S. J.; Rao, C. N. R. arXiv:1009.3700v1. arXiv.org e-Print archive. http:// arxiv.org/abs/1009.3700.

(25) Swathi, R. S.; Sebastian, K. L. J. Chem. Phys. 2009, 130, 086101-086103.

(26) Chen, Z.; Berciaud, S.; Nuckolls, C.; Heinz, T. F.; Brus, L. E. ACS Nano 2010, 4, 2964-2968.

(27) Harrison, B. S.; Atala, A. Biomaterials 2007, 28, 344-353.

(28) Buzsaki, G. Nat. Neuro. 2004, 7, 446-451. 
Figures

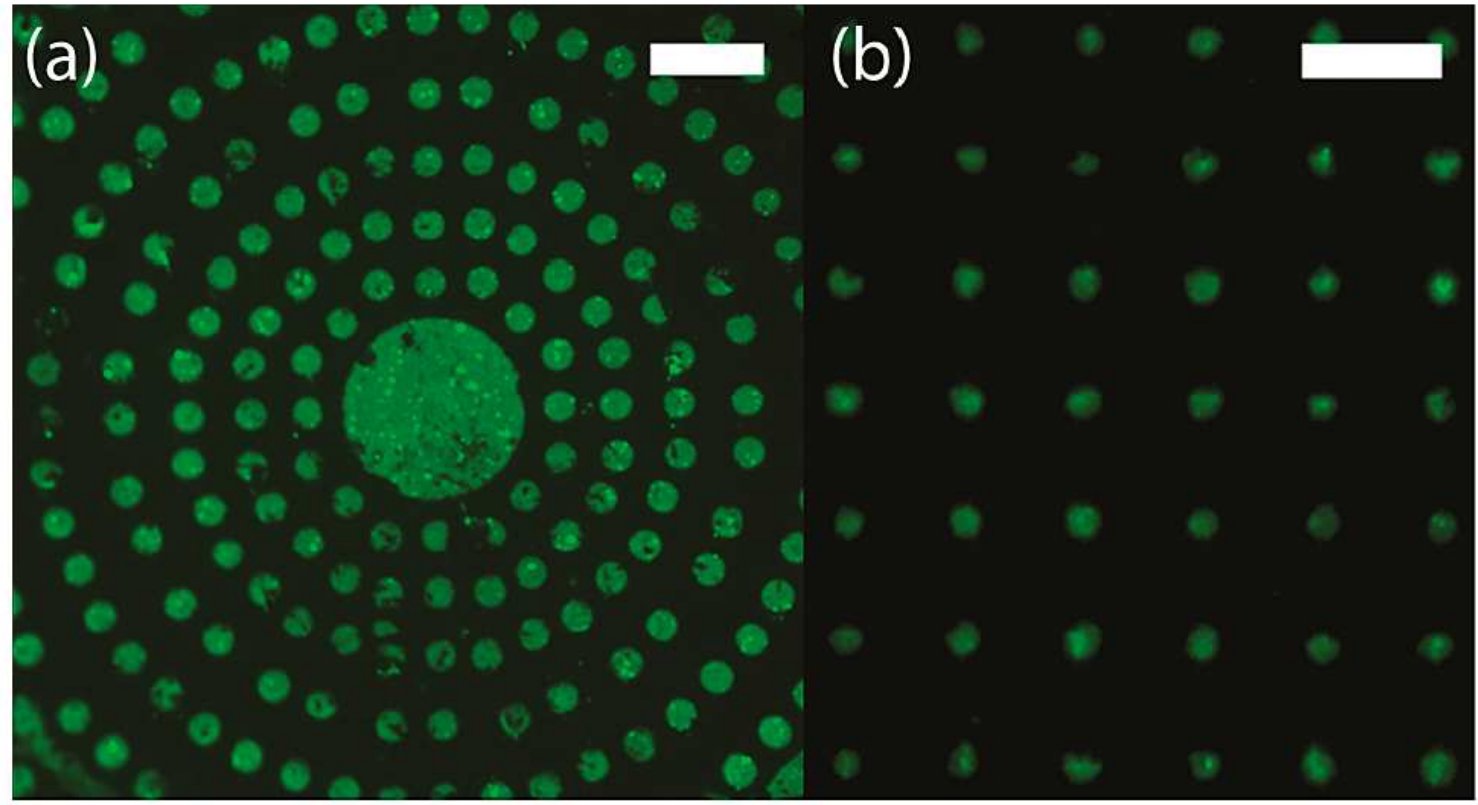

Figure 1. Micropatterned proteins (a) glucose oxidase and (b) laminin on epitaxial graphene treated with PYR-NHS. Scale bars are 20 and $10 \mu \mathrm{m}$, respectively. 

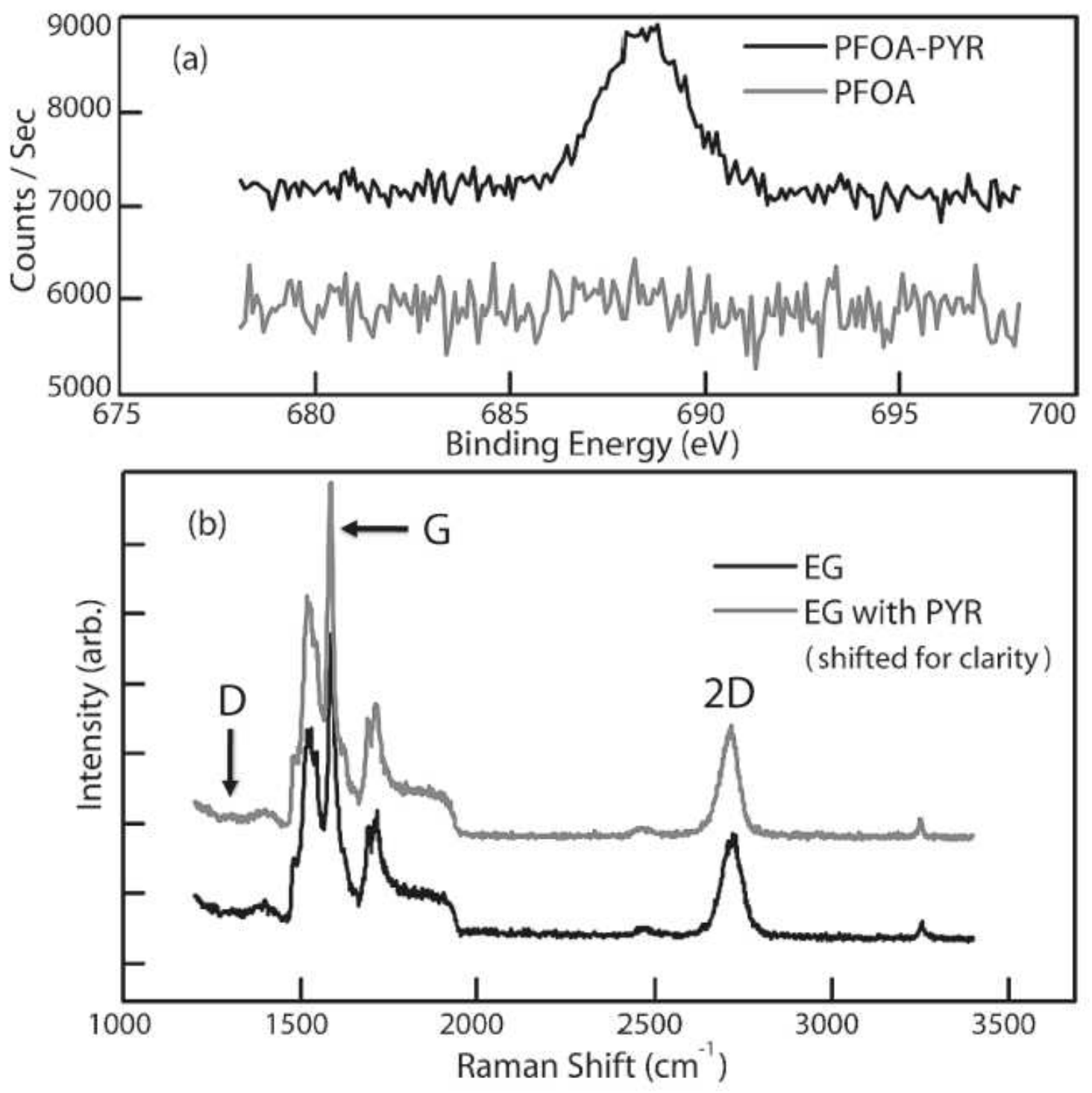

Figure 2. (a) XPS spectra of PFOA-PYR and PFOA-treated epitaxial graphene (EG). Only PFOA-PYRtreated samples have an F 1s peak $(689 \mathrm{eV})$, indicating that PYR irreversibly binds to epitaxial graphene. (b) Raman spectra of PYR-EG and pristine EG. The absence of a D peak at $\sim 1350 \mathrm{~cm}-1$ confirms that the sp2 hybridization remains unperturbed. The 2D Lorentzian peak at $2716 \mathrm{~cm}-1$ before and after treatment indicates that the $\pi$ band remains intact. Non-labeled peaks are $4 \mathrm{H}-\mathrm{SiC}$. 


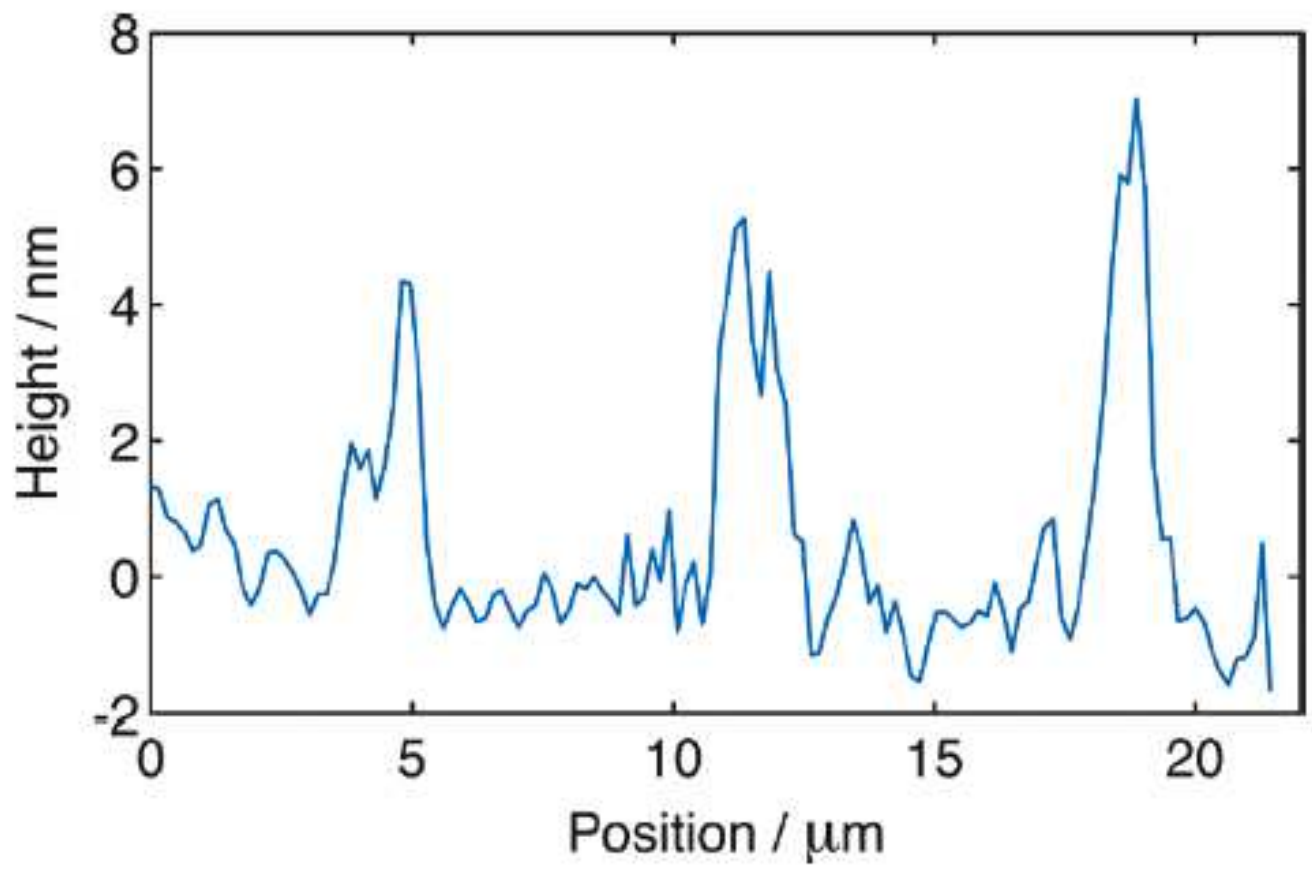

Figure 3. Topographical line profile of three protein areas located on a micropatterned array of glucose oxidase on PYR-treated graphene. 